\title{
On quantum phase crossovers in finite systems
}

\author{
Clare Dunning $\S^{*}$, Katrina E. Hibberd $\boldsymbol{\Upsilon}^{\dagger}$ and Jon Links $\Upsilon^{\ddagger}$ \\ §Institute of Mathematics, Statistics and Actuarial Science, \\ The University of Kent, U.K. \\ ФCentre for Mathematical Physics, School of Physical Sciences, \\ The University of Queensland, Brisbane 4072, Australia.
}

February 1, 2008

\begin{abstract}
In this work we define a formal notion of a quantum phase crossover for certain Bethe ansatz solvable models. The approach we adopt exploits an exact mapping of the spectrum of a many-body integrable system, which admits an exact Bethe ansatz solution, into the quasi-exactly solvable spectrum of a one-body Schrödinger operator. Bifurcations of the minima for the potential of the Schrödinger operator determine the crossover couplings. By considering the behaviour of particular ground-state correlation functions, these may be identified as quantum phase crossovers in the many-body integrable system with finite particle number. In this approach the existence of the quantum phase crossover is not dependent on the existence of a thermodynamic limit, rendering applications to finite systems feasible. We study two examples of bosonic Hamiltonians which admit second-order crossovers.
\end{abstract}

Keywords: quantum integrability (Bethe ansatz), Bose-Einstein condensation (theory)

\section{Introduction}

Quantum phase transitions may occur in the ground state (i.e. at zero temperature) of quantum systems as an interaction coupling is varied. Such phase transitions may be thought of as being driven by quantum fluctuations, in analogy with thermal fluctuations underpinning thermal phase transitions. In fact in many cases there is a correspondence between a quantum phase transition in $d$ dimensions and a thermal phase transition in $(d+1)$ dimensions [1]. One of the important mathematical tools in the study of thermal phase transitions is the thermodynamic limit, where the number of particles is taken to infinity. In this limit phase transitions can be associated with discontinuities in certain physical quantities derived from the free energy.

For finite (i.e. mesoscopic) systems we cannot appeal to this notion of discontinuities to characterise a sharp change between quantum phases, as the transitions are smooth.

*email: t.c.dunning@kent.ac.uk

†email: keh@maths.uq.edu.au

‡email: jrl@maths.uq.edu.au 
Nonetheless for many finite systems there is a sense of a crossover between different quantum phases, and it useful to be able to characterise such a crossover, beyond saying that it is what occurs in a finite system in cases where there is phase transition in the thermodynamic limit. Quantum phases in finite systems have previously been studied in [2] in the context of the Interacting Boson Model (IBM). There it was argued that quantum phase transitions can be identified via "shape transitions" of an effective potential energy surface, defined in terms of classical variables, which becomes exact in the thermodynamic limit. Our goal here is to formulate an approach which entirely avoids the use of the thermodynamic limit, for applications to cases where taking this limit may not be desired or justified. For example there are known models where crossovers between ground-state phases only exist in the finite case. One possible situation is that the boundaries in parameter space between such ground-state phases may merge together as the thermodynamic limit is approached. An example of this is seen in the classical fieldtheoretic analysis of the attractive non-linear Schrödinger equation, where the transition coupling between the uniform regime and the broken symmetry soliton regime occurs at a coupling which scales as $N^{-1}$ for large $N$, where $N$ is the number of particles [3]. In the thermodynamic limit the transition coupling degenerates to zero (free-field case). For the Dicke model there are several "critical points" which degenerate in the thermodynamic limit [4]. Another problematic scenario arises if taking a particular limit of a coupling parameter does not commute with taking the thermodynamic limit, as is known to happen in the weak coupling regimes of the BCS model [5, 6] and the Bose gas with delta function interactions [7]. Moreover for some systems, such as the attractive case of the Bose gas, there are added technical difficulties in defining the thermodynamic limit, as the ground-state energy per particle is not finite in this limit [7, 8].

Motivated by the above considerations, below we give a characterisation for a quantum phase crossover (QPC) in a one-body system with an external potential, for which there is no notion of a thermodynamic limit. Our definition for a QPC is given in terms of properties of the corresponding classical system. We then illustrate how this result can be used to investigate QPCs in Bethe ansatz solvable many-body interacting systems with finite particle number, through a manner which avoids taking the thermodynamic limit. The key to this approach, as will be detailed below, is to exploit the Bethe ansatz solution to perform an exact mapping from the spectrum of the many-body interacting system into the spectrum of a one-body system in a potential.

\section{Quantum phase crossovers}

\section{$2.1 \quad$ One-body systems}

We start with the Schrödinger operator (SO) eigenvalue equation in one dimension

$$
-\frac{\partial^{2} \psi_{k}}{\partial x^{2}}+V(x) \psi_{k}=E_{k} \psi_{k}
$$

where for the potential $V(x)$ it is assumed there is a bifurcation of the global minimum at $x_{0}$, such that we may consider the approximation

$$
V(x) \approx V_{0}-2 V_{1}\left(x-x_{0}\right)^{2}+V_{2}\left(x-x_{0}\right)^{4}
$$

with $V_{2}>0$. A classical treatment of the above is equivalent to Landau theory, as used in the study of thermodynamic phase transitions. This approach has been previously 
discussed in [2]. The classical ground-state energy $\tilde{E}_{0}$ is given, to leading order in $V_{1}$, by

$$
\tilde{E}_{0} \sim\left\{\begin{array}{cl}
V_{0} & \text { for } V_{1}<0 \\
V_{0}-V_{1}^{2} / V_{2} & \text { for } V_{1}>0
\end{array}\right.
$$

Using this result as an approximation to the ground-state energy for the quantum system, we can appeal to the Hellmann-Feynman theorem to approximate the ground-state position fluctuations:

$$
\left\langle\left(x-x_{0}\right)^{2}\right\rangle=-\frac{1}{2} \frac{\partial \tilde{E}_{0}}{\partial V_{1}} \sim\left\{\begin{array}{cc}
0 & \text { for } V_{1}<0 \\
V_{1} / V_{2} & \text { for } V_{1}>0 .
\end{array}\right.
$$

In a full quantum analysis we must expect that quantum fluctuations will smooth out the discontinuity in the derivative of $\left\langle\left(x-x_{0}\right)^{2}\right\rangle$ (and in particular $\left\langle\left(x-x_{0}\right)^{2}\right\rangle>0$ for all $\left.V_{1}\right)$, so there is no quantum phase transition in the traditional sense. Nonetheless, the above classical analysis indicates that around the crossover coupling $V_{1}=0$ we should expect a sharp change in the behaviour of $\left\langle\left(x-x_{0}\right)^{2}\right\rangle$ indicative of a crossover between a localised state and a Schrödinger cat state. Therefore we formally define a QPC for a one-dimensional SO as follows:

Definition 1 Consider the SO equation (1) where the potential $V(x)$ smoothly depends on a dimensionless coupling parameter $\gamma$. Treating (1) as a classical problem, approximate the ground-state energy as the minimum of the potential via $\tilde{E}_{0}=\min _{x \in \mathbb{R}} V(x)$. If $m$ is the smallest integer for which $\partial^{m} \tilde{E}_{0} / \partial \gamma^{m}$ is discontinuous at some coupling $\gamma_{c}$, we say there is an mth-order QPC of the quantum system at $\gamma_{c}$.

Of interest to the examples considered below are second-order crossovers, for which $\partial^{2} \tilde{E}_{0} / \partial \gamma^{2}$ is discontinuous.

\section{$2.2 \quad$ Finite many-body systems}

In the thermodynamic limit there are known examples for which there is a one-to-one correspondence, established via the Bethe ansatz, between the spectrum of an integrable model (IM) and the spectrum of a SO [9, 10, 11]. For IMs acting on finite-dimensional Hilbert spaces an analogous scenario can hold, where the spectrum of the IM maps into the spectrum of a SO, the so-called quasi-exactly solvable (QES) sector [12, 13]. Such a mapping is obviously not one-to-one, but typically the QES spectrum of the SO corresponds to low lying energy levels. In certain cases, such as the examples studied below, the mapping is faithful between the ground-state energies of the IM and the SO. This fact provides the means to explore QPCs in the IM.

Let $H=H(\gamma)$ denote the Hamiltonian for some IM acting on a finite-dimensional Hilbert space where $\gamma$ is a dimensionless coupling parameter. Let $\left|\Psi_{j}\right\rangle$ denote the eigenstates of $H$ with energy levels $\mathcal{E}_{j}$ and the convention that $\mathcal{E}_{0}$ is the ground-state energy. Now assume that the energies $\mathcal{E}_{j}$ can be mapped to energies $E_{k}$ of a SO such that for each $j$ there exists a $k$ satisfying

$$
\mathcal{E}_{j}=\chi E_{k}
$$

for some positive scale factor $\chi$ which is independent of $\gamma, j$ and $k$. Since the mapping of the spectrum of the IM to the corresponding SO is not one-to-one, it is important to assert that the mapping is faithful between the ground-state energies. In our examples, following the arguments in [12, 13], this will be guarranteed by the following oscillation theorem [14]: 
Theorem 1 Consider a SO with locally bounded potential $V(x)$ satisfying $V(x) \rightarrow \infty$ as $|x| \rightarrow \infty$. Let $\psi_{k}, k=0,1, \ldots \infty$ denote the eigenfunctions of the $S O$ with eigenvalues $E_{k}$ respectively, ordered such that $E_{j}<E_{k}$ whenever $j<k$. Then $\psi_{k}$ has precisely $k$ (real) zeroes.

A corollary of the theorem is that the ground state wavefunction of the SO has no zeroes.

Now assume that the mapping (3) does hold between the ground state energies. Defining the operator $\mathcal{A}=\partial H / \partial \gamma$ which acts on the Hilbert space of the IM, we again use the Hellmann-Feynman theorem to deduce that for the ground state

$$
\langle\mathcal{A}\rangle=\frac{\partial \mathcal{E}_{0}}{\partial \gamma}=\chi \frac{\partial E_{0}}{\partial \gamma} \approx \chi \frac{\partial \tilde{E}_{0}}{\partial \gamma} .
$$

Thus the behaviour of the ground-state correlation function $\langle\mathcal{A}\rangle$ will display a sharp change if $\partial^{2} \tilde{E}_{0} / \partial \gamma^{2}$ is discontinuous. This leads to

Definition 2 Suppose the ground state energy $\mathcal{E}_{0}$ of an IM exactly maps to the ground state energy $E_{0}$ of a SO through (3). If the $S O$ exhibits a second-order QPC at some dimensionless coupling $\gamma_{c}$ as in Definition 1, then we say that the IM also exhibits a second-order $Q P C$ at $\gamma_{c}$.

Having outlined the theroetical considerations, we now apply it to specific models.

\section{Examples}

\subsection{Atomic-molecular bosonic model}

The first model we look at describes the interconversion of bosonic atomic and di-atomic molecular modes [15]. The Hamiltonian is

$$
H=\frac{\delta}{2} n_{a}+\frac{\Omega}{2}\left(a^{\dagger} a^{\dagger} b+b^{\dagger} a a\right)
$$

where $a^{\dagger}$ and $b^{\dagger}$ denote the creation operators for atomic and molecular modes respectively and as usual $n_{a}=a^{\dagger} a, n_{b}=b^{\dagger} b$. The total particle number $N=n_{a}+2 n_{b}$ is conserved. In addition the Hamiltonian is invariant under the transformation $\left(a, a^{\dagger}\right) \rightarrow\left(-a,-a^{\dagger}\right)$. Since the change $\Omega \rightarrow-\Omega$ is equivalent to the unitary transformation $\left(b, b^{\dagger}\right) \rightarrow\left(-b,-b^{\dagger}\right)$ we restrict to $\Omega>0$.

The Hamiltonian admits an exact Bethe ansatz solution [16]. The solution depends on a discrete variable $p$ which takes values 0 or 1 , depending on whether the total particle number is even or odd. The exact solution gives the energy levels as

$$
E=\delta\left(M+\frac{p}{2}\right)+\Omega \sum_{j=1}^{M} v_{j}
$$

where the parameters $\left\{v_{j}\right\}$ are the roots of the Bethe ansatz equations

$$
\frac{2 p+1}{2 v_{j}}-v_{j}-\gamma=\sum_{k \neq j}^{M} \frac{2}{v_{k}-v_{j}} \quad j=1, \ldots, M .
$$

Above, $\gamma=\delta / \Omega$ is defined as the dimensionless coupling, the number of roots $M$ is related to the total particle number through $N=2 M+p$, and the dimension of the Hilbert space 


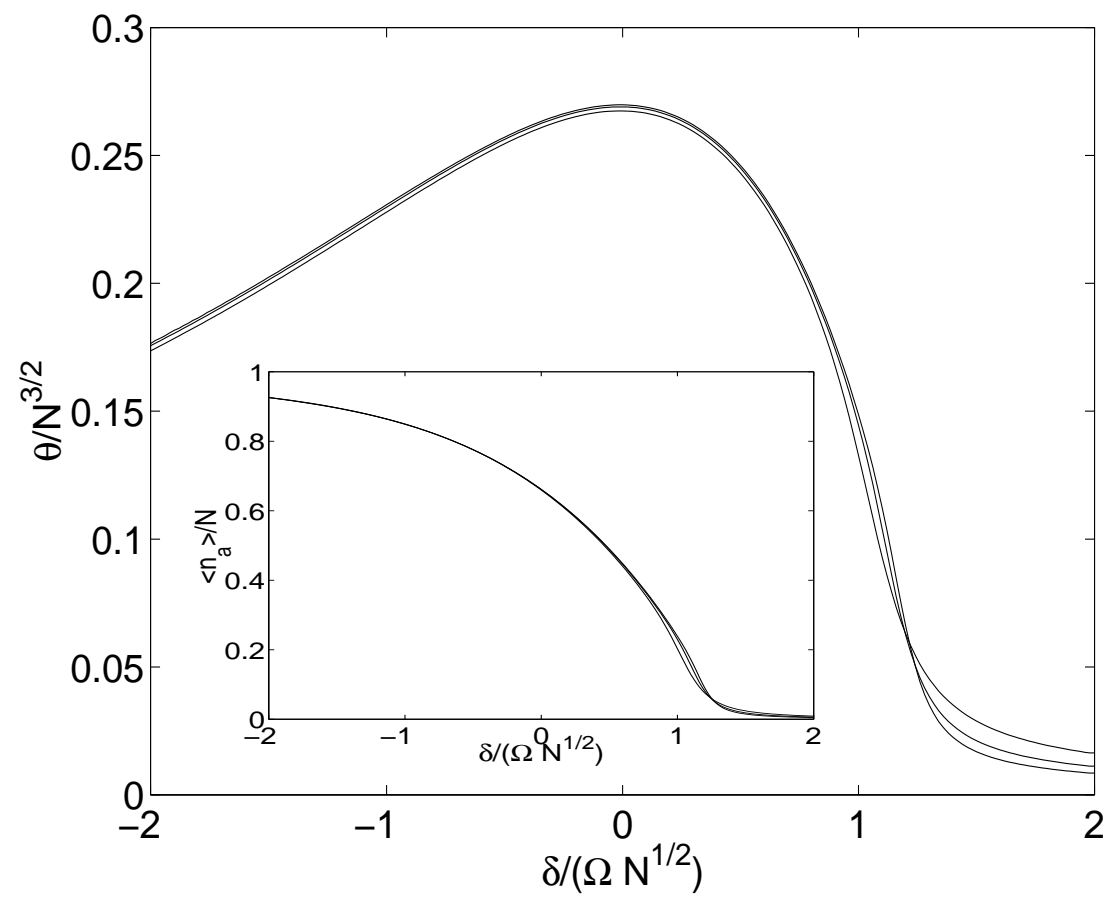

Figure 1: Behaviour of the coherence correlator (7) for the ground state of (44). The curves shown are for total particle number $N=20,30,40$. The inset shows $\left\langle n_{a}\right\rangle / N$, the average fractional occupation of unbound atoms in the ground state. Below the crossover coupling $\delta /(\Omega \sqrt{N}) \approx 1.4$ the ground state is a coherent superposition of atomic and molecular states, while above the crossover coupling the ground state predominantly consists of molecular bosons.

is $M+1$. From the ground-state energy, one can use the Hellman-Feynman theorem to compute the ground-state correlations

$$
\left\langle n_{a}\right\rangle=2 \frac{\partial \mathcal{E}_{0}}{\partial \delta}, \quad \theta=-\frac{\partial \mathcal{E}_{0}}{\partial \Omega}
$$

where

$$
\theta=-\left\langle a^{\dagger} a^{\dagger} b+b^{\dagger} a a\right\rangle / 2
$$

is the coherence correlator.

Results of numerical analysis of the exact solution are shown in Fig. 1, which has been taken from [16]. Despite the very small number of particles, it can be deduced that below the crossover coupling $\delta /(\Omega \sqrt{N}) \approx 1.4$ the ground state is a coherent superposition of atomic and molecular states, while above the crossover coupling the ground state predominantly consists of molecular bosons (see also Fig. 1 of [17]). The crossover sharpens with increasing $N$ but becomes singular in the thermodynamic limit $N \rightarrow \infty$, implying $\Omega \rightarrow 0$ and the eigenstates approach Fock states. However in this limit the ground-state energy per particle is not finite when $\delta / \Omega<0$ (see comments in the Conclusion). We note that the qualitative features of $\left\langle n_{a}\right\rangle / N$ shown in the inset are the same as those shown in Fig. 3 of 2 for the IBM. There it was argued the result could be interpreted as a second-order crossover, even for particle numbers of the order $10^{1}$. For (44) we will show that this same conclusion can be obtained from our approach described above.

To derive the value of the crossover coupling from the Bethe ansatz solution, we map the spectrum into that of a $\mathrm{SO}$ with a sextic potential. The procedure can be found in 


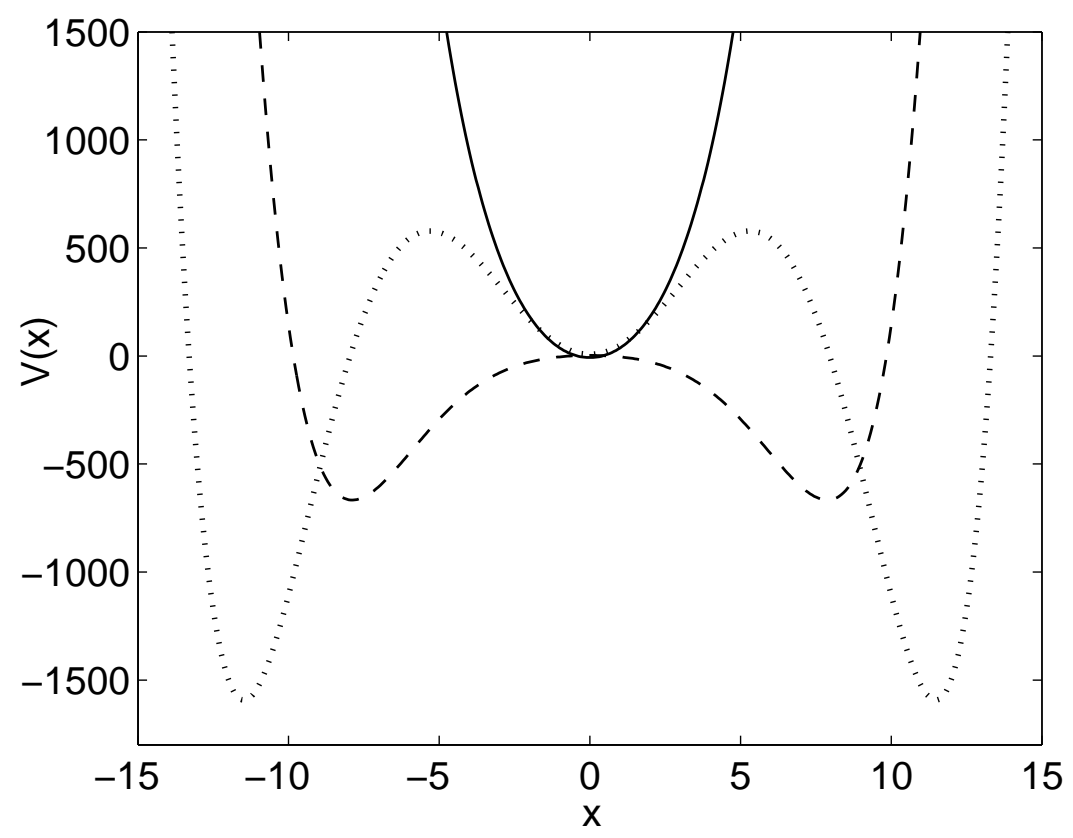

Figure 2: Generic behaviour of the sextic potential (9) for different values of the dimensionless coupling $\gamma$, where the crossover coupling is given by $\gamma_{c}=\sqrt{2 N+3}$. Here we take $N=100$. For $\gamma>\gamma_{c}$ (solid line $\left.-\gamma=30\right)$ there is a single minimum at $x=0$. For $-\gamma_{c}<\gamma<\gamma_{c}$ (dash line $\left.-\gamma=-10\right)$ or $\gamma<-\gamma_{c}$ (dot line $\left.-\gamma=-30\right)$ there are two global minima at $\pm x_{0}$ given by (10).

[12], so we simply give the result (see also [13]). For a given solution of (66) with energy given by (5), we set

$$
\psi(x)=x^{p} \exp \left(-\frac{\gamma x^{2}}{8}-\frac{x^{4}}{64}\right) \prod_{j=1}^{M}\left(\frac{x^{2}}{4}-v_{j}\right) .
$$

Then $\psi(x)$ satisfies (1) with $\chi=\Omega$ and potential

$$
V(x)=-\frac{\gamma}{4}+\frac{\left(\gamma^{2}-3-2 N\right) x^{2}}{16}+\frac{\gamma x^{4}}{32}+\frac{x^{6}}{256} .
$$

The number of QES states (8) is the dimension of the Hilbert space for (44), $M+1$. It is apparent that the states (8) are even (odd) functions of $x$, with an even (odd) number of zeroes for $p=0(p=1)$ and at most $2 M(2 M+1)$ zeroes. In view of Theorem 1 no two states can have the same number of zeroes. For $p=0$ the QES states must be the states $\psi_{k}$ such that $k=0,2,4, \ldots, 2 M$. Therefore the ground state of the SO with potential (9) lies in the QES sector, and the mapping of the spectrum of (4) into that of the SO is faithful for the ground-state energies. For a system with odd particle number, the QES states correspond to the $k=1,3, \ldots, 2 M+1$ states of the associated SO. For this case one can alternatively consider the potential to be restricted to the half line $x \geq 0$ with the hard-wall boundary condition $V(0)=\infty$, requiring $\psi(0)=0$. Then the QES states have an even number of zeroes in $(0, \infty)$ and correspond to the states $\psi_{k}$ such that $k=0,2,4, \ldots, 2 M$, which includes the ground state. 
For $\gamma>0$ and $\left(\gamma^{2}-3-2 N\right)>0$ the potential (9) attains its minimum at $x_{0}=0$, while for $\left(\gamma^{2}-3-2 N\right)<0$ or $\gamma<0$ minima occur at

$$
x_{0}^{2}=\frac{4}{3} \sqrt{4 \gamma^{2}-3\left(\gamma^{2}-3-2 N\right)}-\frac{8 \gamma}{3} .
$$

This analysis identifies the crossover coupling as $\gamma_{c}=\sqrt{2 N+3}$. As $\gamma \rightarrow\left(\gamma_{c}\right)_{-}$we find $x_{0} \sim \pm(\sqrt{2 N+3}-\gamma)^{1 / 2}$. The above shows that for the potential (9) there is a secondorder QPC of the same universality class as the Landau (or mean-field) theory, which is also the same class for the second-order crossover of the IBM [2]. In the classical approximation the behaviour of the correlation functions is found to be

$$
\begin{array}{ll}
\theta-\theta_{c} \sim \gamma-\gamma_{c}, & \gamma \rightarrow\left(\gamma_{c}\right)_{-} \\
\theta-\theta_{c} \sim 0, & \gamma \rightarrow\left(\gamma_{c}\right)_{+},
\end{array}
$$

with $\theta_{c}=0$. Similarly results hold for $\left\langle n_{a}\right\rangle$. These are consistent with Fig. 1.

\subsection{Attractive two-site Bose-Hubbard model}

Quantum tunneling of bosons, based on a two-mode approximation, can be described by the two-site Bose-Hubbard Hamiltonian [18]:

$$
H=-\frac{k}{8}\left(n_{1}-n_{2}\right)^{2}-\frac{\mathcal{E}}{2}\left(b_{1}^{\dagger} b_{2}+b_{2}^{\dagger} b_{1}\right)
$$

where $b_{j}^{\dagger}, j=1,2$ denote the single-particle creation operators associated with two bosonic modes and $n_{1}=b_{1}^{\dagger} b_{1}$ and $n_{2}=b_{2}^{\dagger} b_{2}$ are the corresponding number operators. The total particle number $N=n_{1}+n_{2}$ is conserved. We only consider the attractive case for which $k>0$, and take $\mathcal{E}>0$. The case $\mathcal{E}<0$ can be obtained by the unitary transformation $\left(b_{1}, b_{1}^{\dagger}\right) \rightarrow\left(-b_{1},-b_{1}^{\dagger}\right)$.

To derive the Bethe ansatz solution we follow the approach of [13, 19]. We start with the Jordan-Schwinger realisation of the $s u(2)$ algebra generators

$$
S^{+}=b_{1}^{\dagger} b_{2}, \quad S^{-}=b_{2}^{\dagger} b_{1}, \quad S^{z}=\frac{1}{2}\left(N_{1}-N_{2}\right)
$$

which satisfy the commutation relations

$$
\left[S^{z}, S^{ \pm}\right]= \pm S^{ \pm}, \quad\left[S^{+}, S^{-}\right]=2 S^{z}
$$

The realisation is $(N+1)$-dimensional when the constraint of fixed particle number $N=$ $N_{1}+N_{2}$ is imposed. In terms of this realisation the Hamiltonian may be written

$$
H=-\frac{k}{2}\left(S^{z}\right)^{2}-\frac{\mathcal{E}}{2}\left(S^{+}+S^{-}\right)
$$

The same $(N+1)$-dimensional representation of $s u(2)$ is given by the mapping to differential operators

$$
S^{z}=u \frac{\mathrm{d}}{\mathrm{d} u}-\frac{N}{2}, \quad S^{+}=N u-u^{2} \frac{\mathrm{d}}{\mathrm{d} u}, \quad S^{-}=\frac{\mathrm{d}}{\mathrm{d} u}
$$


acting on the $(N+1)$-dimensional space of polynomials with basis $\left\{1, u, u^{2}, \ldots, u^{N}\right\}$. We can then equivalently represent (13) as the second-order differential operator

$$
\begin{aligned}
H & =-\frac{k}{2}\left(u^{2} \frac{\mathrm{d}^{2}}{\mathrm{~d} u^{2}}+(1-N) u \frac{\mathrm{d}}{\mathrm{d} u}+\frac{N^{2}}{4}\right)-\frac{\mathcal{E}}{2}\left(N u+\left(1-u^{2}\right) \frac{\mathrm{d}}{\mathrm{d} u}\right) \\
& =-\frac{k u^{2}}{2} \frac{\mathrm{d}^{2}}{\mathrm{~d} u^{2}}+\frac{1}{2}\left(k(N-1) u-\mathcal{E}\left(1-u^{2}\right)\right) \frac{\mathrm{d}}{\mathrm{d} u}-\frac{k N^{2}}{8}-\frac{\mathcal{E} N u}{2}
\end{aligned}
$$

Solving for the spectrum of the Hamiltonian (11) is then equivalent to solving the eigenvalue equation

$$
H Q=E Q
$$

where $H$ is given by (14) and $Q(u)$ is a polynomial function of $u$ of order $N$. To obtain the Bethe ansatz solution, we first express $Q(u)$ in terms of its roots $\left\{v_{j}\right\}$ :

$$
Q(u)=\prod_{j=1}^{N}\left(u-v_{j}\right)
$$

Evaluating (15) at $u=v_{k}$ for each $k$ leads to the set of Bethe ansatz equations

$$
\frac{\mathcal{E}\left(1-v_{k}^{2}\right)+k(1-N) v_{k}}{k v_{k}^{2}}=\sum_{j \neq k}^{N} \frac{2}{v_{j}-v_{k}}, \quad k=1, \ldots, N
$$

Writing the asymptotic expansion $Q(u) \sim u^{N}-u^{N-1} \sum_{j=1}^{N} v_{j}$ and by considering the terms of order $N$ in (15), the energy eigenvalues are found to be

$$
E=-\frac{k N^{2}}{8}+\frac{\mathcal{E}}{2} \sum_{j=1}^{N} v_{j}
$$

We may exactly map the spectrum of (11) into that of the SO equation (1). Setting $\gamma=\mathcal{E} / k, \chi=k / 2$ and

$$
\psi(x)=\exp (-\gamma \cosh (x)) \prod_{j=1}^{N}\left(\exp \left(\frac{x}{2}\right)-v_{j} \exp \left(\frac{-x}{2}\right)\right)
$$

then, as a result of (15), $\psi(x)$ satisfies (1) with the potential

$$
V(x)=\gamma^{2} \sinh ^{2}(x)-(N+1) \gamma \cosh (x)
$$

whenever the $\left\{v_{j}\right\}$ satisfy (16). Using the same argument as in the previous example, it can be established that this mapping is faithful for the ground-state energies.

The potential (18) is a double Morse potential, which has previously been studied as a quasi-exactly solvable potential [13, 20]. It is a single well potential when $2 \gamma>(N+1)$ with a mimimum at $x_{0}=0$, and a double well for $2 \gamma<(N+1)$ with minima at $x_{0}=$ $\pm \cosh ^{-1}[(N+1) / 2 \gamma]$. We identify the crossover coupling as $\gamma_{c}=(N+1) / 2$, and deduce that as $\gamma \rightarrow\left(\gamma_{c}\right)_{-}$

$$
\pm x_{0} \sim((N+1) / 2-\gamma)^{1 / 2}
$$


establishing that (11) is in the same universality class as (41). The coherence correlator in this instance, $\theta=-\left\langle b_{1}^{\dagger} b_{2}+b_{2}^{\dagger} b_{1}\right\rangle / 2$, displays the classical critical behaviour

$$
\begin{array}{lll}
\theta-\theta_{c} \sim \gamma-\gamma_{c}, & \gamma \rightarrow\left(\gamma_{c}\right)_{-} \\
\theta-\theta_{c} \sim 0, & \gamma \rightarrow\left(\gamma_{c}\right)_{+}
\end{array}
$$

where $\theta_{c}=k \gamma_{c}$. We note that (18), with change of variable $x \rightarrow i x$, was previously derived in [21] as an exact quantum phase model for the repulsive case of (11).

The QPC coupling $\gamma_{c}=(N+1) / 2$ agrees, to leading order in $N$, with the results of [22]. In that work the QPC arises as the onset of broken symmetry in the mean-field analysis. The result is also in agreement with [23], where the QPC was identified by a numerical analysis of wave function overlaps (cf. [24]) and ground-state entanglement measured by the von Neumann entropy. In particular it was found that there was a peak in the ground-state entanglement at the crossover coupling. This is consistent with the claim of [25] that a peak in the ground-state entanglement occurs when there is a supercritical pitchfork bifurcation of the global minimum of the Hamiltonian in the phase space of the semi-classical limit. For (11), the semi-classical dynamics have been studied in [26] and it is seen from the equations of motion that such a bifurcation does occur at $\gamma_{c}$ (to leading order). It is interesting to note that in contrast there is no peak in the entanglement at $\gamma_{c}$ for the Hamiltonian (41) [27]. For this latter case it has been shown in [28] that the bifurcation occuring in the semi-classical phase space is not of the supercritical pitchfork type.

\section{Conclusion}

We have developed a technique for determining QPCs in certain finite IMs admitting a Bethe ansatz solution, and applied it to the Hamiltonians (4]11). In both cases we identified a dimensionless crossover coupling $\gamma_{c}$, whose role in defining the QPC is supported by the behaviour of particular ground-state correlation functions. The significance of the results is reflected by the fact that in both cases $\gamma_{c}$ is $N$-dependent, which highlights the potential difficulties in studying quantum phases in the thermodynamic limit. Furthermore, for the Hamiltonian (44) the ground state energy per particle $\mathcal{E}_{0} / N$ scales as $N^{1 / 2}$ whenever $\gamma<0$ (as can be deduced from equations (9) and (10)). For the Hamiltonian (11) the ground-state energy per particle at $\gamma_{c}$ is given, in the classical approximation, by $\mathcal{E}_{0} / N=k N(1+1 / N)^{2} / 4$. In both cases the ground-state energy per particle is not finite in the thermodynamic limit.

Both models we have studied have only two degrees of freedom. In generic bosonic systems it is quite reasonable for a system to have a low number of degrees of freedom, since the bosonic statistics allow for arbitrarily high particle number independent of the degrees of freedom. This situation is in stark contrast to fermionic systems where, due to the exclusion principle, increasing the number of particles is associated with increasing the number of degrees of freedom when taking the thermodynamic limit. A consequence of this difference between bosonic and fermionic systems is that for bosonic systems with small number of degrees of freedom, such as the two studied here, it is not possible to apply renormalisation group techniques to determine the nature of the ground-state phases.

Finally, we comment on the feasability of extensions to other models. Mappings from the spectrum of a many-body Hamiltonian to an SO can be constructed in cases where there is a spectrum generating Lie algebra such that the Hamiltonian commutes with the Casimir invariants. The $s u(2)$ case has been discussed in [20], where it was argued that 
31 classes of quasi-exactly SOs exist. Other examples can be found in [13]. However it remains a challenge to determine the full extent of applicability of the methods we have introduced here.

\section{Acknowledgement}

We gratefully acknowledge financial support from the Australian Research Council through the Discovery Project DP0557949.

\section{References}

[1] Sachdev S, 1999 Quantum phase transitions (Cambridge University Press)

[2] Iachello F and Zamfir N V, Quantum phase transitions in mesoscopic systems, 2004 Phys. Rev. Lett. 92212501

[3] Kanamoto R, Saito H, and Ueda M, Quantum phase transition in one-dimensional Bose-Einstein condensates with attractive interactions, 2003 Phys. Rev. A 67013608

[4] Bužek V, Orszag M, and Roško M, Instability and entanglement of the ground state of the Dicke model, 2005 Phys. Rev. Lett. 94163601

[5] Schechter M, Imry Y, Levinson Y, and von Delft J, Thermodynamic properties of a small superconducting grain, 2001 Phys. Rev. B 63214518

[6] Dunning C, Links J, and Zhou H-Q, Ground-state entanglement of the BCS model, 2005 Phys. Rev. Lett. 94227002

[7] Batchelor M T, Bortz M, Guan X W, and Oelkers N, Evidence for the super TonksGirardeau gas, 2005 J. Stat. Mech.: Theor. Exp. L10001

[8] McGuire J B, Study of exactly soluble one-dimensional N-body problems, $1964 \mathrm{~J}$. Math. Phys. 5622

[9] Dorey P and Tateo R, Anharmonic oscillators, the thermodynamic Bethe ansatz and nonlinear integral equations, 1999 J. Phys. A: Math. Gen. 32 L419

[10] Dorey P, Dunning C, and Tateo R, Spectral equivalences, Bethe ansatz equations, and reality properties in $\mathcal{P}$ T-symmetric quantum mechanics $2001 \mathrm{~J}$. Phys. A: Math. Gen. 345679

[11] Bazhanov V V, Lukyanov S L, and Zamolodchikov A B, Spectral determinants for Schrödinger equation and Q-operators of conformal field theory, 2001 J. Stat. Phys. 102567

[12] Ushveridze A G, 1994 Quasi-exactly solvable models in quantum mechanics (Institute of Physics Publishing, Bristol and Philadelphia)

[13] Ulyanov V V and Zaslavskii O B, New methods in the theory of quantum spin systems, 1992 Phys. Rep. 216179 
[14] Berezin F A and Shubin M A, 1991 The Schrödinger equation (Kluwer Academic Publishers, Dordrecht)

[15] Vardi A, Yurovsky V A, and Anglin J R, Quantum effects on the dynamics of a two-mode atom-molecule Bose-Einstein condensate, 2001 Phys. Rev. A 64063611

[16] Zhou H-Q, Links J, and McKenzie R H, Exact solution, scaling behaviour and quantum dynamics of a model of an atom-molecule Bose-Einstein condensate, 2003 Int. J. Mod. Phys. B 175819

[17] Koštrun M, Mackie M, Côté R, and Javanainen J, Theory of coherent photoassociation of a Bose-Einstein condensate, 2000 Phys. Rev. A 62063616

[18] Leggett A J, Bose-Einstein condensation in the alkali gases: Some fundamental concepts, 2001 Rev. Mod. Phys. 73307

[19] Enol'skii V Z, Kuznetsov V B, and Salerno M, On the quantum inverse scattering method for the DST dimer, 1993 Physica D 68138

[20] Konwent H, Machnikowski P, Magnuszewski P, and Radosz A, Some properties of double Morse potentials, 1998 J. Phys. A: Math. Gen. 317541

[21] Anglin J R, Drummond P, and Smerzi A, Exact quantum phase model for mesoscopic Josephson junctions, 2001 Phys. Rev. A 64063605

[22] Cirac J I, Lewenstein M, Molmer K, and Zoller P, Quantum superposition states of Bose-Einstein condensates, 1998 Phys. Rev. A 571208

[23] Pan F and Draayer J P, Quantum critical behavior of two coupled Bose-Einstein condensates, 2005 Phys. Lett. A 339403

[24] Zanardi P and Paunković N, Ground state overlap and quantum phase transitions, 2006 Phys. Rev. E $\mathbf{7 4} 31123$

[25] Hines A P, McKenzie R H, and Milburn G J, Quantum entanglement and fixed-point bifurcations, 2005 Phys. Rev. A 71042303

[26] Kohler S and Sols F, Oscillatory decay of a two-component Bose-Einstein condensate, 2002 Phys. Rev. Lett. 89060403

[27] Hines A P, McKenzie R H, and Milburn G J, Entanglement of two-mode BoseEinstein condensates, 2003 Phys. Rev. A 67013609

[28] Santos G, Tonel A, Foerster A, and Links J, Classical and quantum dynamics of a model for atomic-molecular Bose-Einstein condensates, 2006 Phys. Rev. A 73023609 